\title{
Quantitation of nitrofurantoin in human plasma by liquid chromatography tandem mass spectrometry
}

\author{
DINESH S. PATEL ${ }^{1,2}$ \\ NAVEEN SHARMA ${ }^{2}$ \\ MUKESH C. PATEL ${ }^{1}$ \\ BHAVIN N. PATEL $2,3 *$ \\ PRANAV S. SHRIVASTAV $3 *$ \\ MALLIKA SANYAL ${ }^{4}$ \\ ${ }^{1}$ Chemistry Department \\ Pramukh Swami Science and \\ H.D. Patel Arts College \\ Sarva Vidyalaya Campus \\ Kadi-382715, Gujarat, India \\ 2 Bio-Analytical Laboratory \\ Cliantha Research Ltd., Bodakdev \\ Ahmedabad-380054, Gujarat, India \\ ${ }^{3}$ Department of Chemistry \\ School of Sciences, Gujarat University \\ Navrangpura, Ahmedabad-380009 \\ Gujarat, India \\ ${ }^{4}$ Department of Chemistry \\ St. Xavier's College, Navrangpura \\ Ahmedabad-380009, Gujarat, India
}

\begin{abstract}
A reliable, selective and sensitive LC-MS/MS assay has been proposed for the determination of nitrofurantoin in human plasma. The analyte and nitrofurazone were extracted from $100 \mu \mathrm{L}$ of human plasma via SPE on Strata-X $33 \mu \mathrm{m}$ extraction cartridges. Chromatography was done on a BDS Hypersil C18 (100 mm $\times 4.6 \mathrm{~mm}, 5 \mu \mathrm{m})$ column under isocratic conditions. Quantitation was done using the multiple reaction monitoring (MRM) mode for deprotonated precursor to product ion transitions of nitrofurantoin $(\mathrm{m} / \mathrm{z} 237.0 \rightarrow 151.8)$ and nitrofurazone $(\mathrm{m} / \mathrm{z}$ $197.0 \rightarrow 123.9)$. The limit of detection and the lowest limit of quantitation of the method were $0.25 \mathrm{ng} \mathrm{mL}^{-1}$ and $5.00 \mathrm{ng} \mathrm{mL} \mathrm{m}^{-1}$, respectively, with a linear dynamic range of 5.00-1500 $\mathrm{ng} \mathrm{mL}^{-1}$ for nitrofurantoin. The intra-batch and inter-batch precision (RSD, \%) was $\leq 5.8 \%$, while the mean extraction recovery was $>92 \%$. The method was successfully applied to a bioequivalence study of a $100 \mathrm{mg}$ nitrofurantoin capsule formulation in 36 healthy subjects.
\end{abstract}

Keywords: nitrofurantoin, LC-MS/MS, solid phase extraction, human plasma, bioequivalence, incurred sample reanalysis

Nitrofurans belong to a group of synthetic broad spectrum antibacterial agents having a nitrofuran ring. Nitrofurantoin [NFT, 1-\{(5-nitro-2-furyl)methylideneamino $\}$ imidazolidine-2,4-dione] is a nitro furan-derivative antibacterial agent, used in human and veterinary medicine $(1,2)$. In humans, it is widely used to treat urinary tract infections. NFT is effective against common urinary tract pathogens, including Escherichia coli, Enterococci, Kleb-

\footnotetext{
*Correspondence; e-mails: pranav_shrivastav@yahoo.com; bhavinpatel27@rediffmail.com
} 
siella and Enterobacter. In therapeutic doses, it achieves urinary levels of $>200 \mu \mathrm{g} \mathrm{mL}{ }^{-1}$. It is generally bacteriostatic at low concentrations $\left(5-10 \mu \mathrm{g} \mathrm{mL}^{-1}\right)$ and bactericidal at higher concentrations $(3,4)$. After oral administration, NFT is well absorbed from the gastrointestinal tract, with most of the absorption taking place in the proximal small intestine (5). The drug is $20-60 \%$ plasma protein bound and its bioavailability is affected by ingestion of food, particle size of the drug formulation and $\mathrm{pH}$. It has been reported that bioavailability of the drug increases from 87 (under fasting) to $94 \%$ when taken with food (6). The plasma half-life of NFT is $\sim 30 \mathrm{~min}$ and is partially metabolized in the liver to form amino-nitrofurantoin. After oral administration, 30-50 \% NFT is excreted intact in urine and $\sim 1 \%$ is excreted in urine as amino-nitrofurantoin (4).

Several analytical methods capable of determining NFT in biological matrices have been reported based on stripping voltammetry $(7,8)$, high performance liquid chromatography (9-12) and LC-MS/MS (13, 14). These procedures have employed protein precipitation (PP) $(12,13)$, liquid-liquid extraction (LLE) $(10,14)$ or solid phase extraction (SPE) (11) for quantitative and consistent recovery of NFT from human plasma. In all chromatographic methods, the sensitivity $(9-14)$, analysis time $(11,12)$ or plasma volume for processing $(11,12)$ were the limiting factor. A selective HPLC-UV method has been developed by Muth et al. (10) for determination of NFT in human plasma and urine. The limit of quantification in plasma and urine was 10 and $380 \mathrm{ng} \mathrm{mL}^{-1}$, respectively, while the chromatographic run time was $15 \mathrm{~min}$. Adkison et al. (13) studied the effect of oral NFT pharmacokinetics in healthy subjects by developing an LC-MS/MS method. The plasma samples were prepared by protein precipitation and the calibration range was 20-10000 ng mL mL $^{-1}$ Recently, Patil and co-workers (14) developed an LC-MS/MS method with the limit of quantitation of $10.2 \mathrm{ng} \mathrm{mL}^{-1}$; however, the application was not reported.

In the present study, a sensitive, selective, robust and rapid LC-MS/MS method has been proposed for the determination of NFT in human plasma. The method is extensively validated as per official guidelines (15) and should be sensitive enough for routine subject sample analysis.

\section{EXPERIMENTAL}

\section{Chemicals and materials}

Reference standard material of nitrofurantoin (NFT, $99.9 \%$ ) and nitrofurazone (IS, $99.5 \%$ ) was procured from the USP Reference Standard (U.S. Pharmacopeial Convention, USA). HPLC grade methanol, acetonitrile, analytical grade reagent ammonium acetate $(98.0 \%)$ and ammonia solution (30.0 \%) were obtained from S.D. Fine Chemicals Ltd. (India). HPLC grade dimethyl formamide (DMF) was obtained from Spectro Chem Pvt. Ltd., (India). Strata-X polymeric reversed phase having $33 \mu \mathrm{m}$ particle size and $800 \mathrm{~m}^{2}$ $\mathrm{g}^{-1}$ surface area $\left(1 \mathrm{~cm}^{3}, 30 \mathrm{mg}\right)$ extraction cartridges were obtained from Phenomenex (USA). Water used for LC-MS/MS was prepared using an Milli Q water purification system from Millipore (India). Control buffered $\left(\mathrm{K}_{3} \mathrm{EDTA}\right)$ human plasma was procured from the Clinical Department, Cliantha Research Limited (India), and was stored at $-20{ }^{\circ} \mathrm{C}$. 


\section{LC-MS/MS instrumentation and conditions}

The liquid chromatography system from Shimadzu (Japan) consisted of a LC-10ADvp pump, an autosampler (SIL-HTc) and an on-line degasser (DGU-14A). Chromatographic column used was BDS Hypersil C18 $(100 \mathrm{~mm} \times 4.6 \mathrm{~mm}, 5.0 \mu \mathrm{m})$ from Thermo Scientific (USA). For isocratic elution, the mobile phase consisted of acetonitrile $/ 0.25 \mathrm{mmol} \mathrm{L}^{-1} \mathrm{am}-$ monium acetate $(60: 40, V / V)$ at a flow rate of $0.5 \mathrm{~mL} \mathrm{~min}^{-1}$. The auto sampler temperature was maintained at $4{ }^{\circ} \mathrm{C}$ and the injection volume was kept at $5.0 \mu \mathrm{L}$. Ionization and detection of analyte and IS were performed on a triple quadrupole mass spectrometer, API-3000 equipped with Turbo Ion spray ${ }^{\circledR}$, from MDS SCIEX (Canada) operating in the negative ion mode. Quantitation was done using the multiple reaction monitoring (MRM) mode to monitor deprotonated precursor $\rightarrow$ product ion transition of $m / z 237.0 \rightarrow 151.8$ for NFT and $197.0 \rightarrow 123.9$ for IS. All the LC and MS parameters were controlled by Analyst software version 1.4.2.

For NFT and IS, the source dependant parameters maintained were gas 1 (nebulizer gas) $68947 \mathrm{~Pa}$, ion spray voltage $-4500 \mathrm{~V}$, turbo heater temperature $475{ }^{\circ} \mathrm{C}$, entrance potential $-10 \mathrm{~V}$, collision activation dissociation (CAD) $41368 \mathrm{~Pa}$, curtain gas $41368 \mathrm{~Pa}$. The compound dependent parameters such as declustering potential, focusing potential, collision energy and cell exit potential were optimized at $-29,-160,-17$ and $-13.5 \mathrm{~V}$ for NFT and $-30,-175,-15$ and $-11 \mathrm{~V}$ for IS, respectively. Quadrupole 1 and 3 were maintained at unit resolution, while the dwell time was set at $300 \mathrm{~ms}$ for both NFT and IS.

\section{Preparation of standard stock and plasma samples}

The NFT standard stock solution of $500 \mu \mathrm{g} \mathrm{mL} \mathrm{m}^{-1}$ was prepared by dissolving the required amount in DMF/deionized water $(50: 50, V / V)$. This was further diluted in the same diluent to get an intermediate solution of $30 \mu \mathrm{g} \mathrm{mL}^{-1}$. Working solutions of NFT for spiking plasma calibration and quality control samples were subsequently prepared using the standard and intermediate stock solutions. The IS stock solution of $100 \mu \mathrm{g} \mathrm{mL}-1$ was prepared by dissolving the needed amount of nitrofurazone in DMF/deionized water (50:50, $V / V)$. IS working solution $\left(1000 \mathrm{ng} \mathrm{mL}^{-1}\right)$ was prepared from the stock solution in deionized water. All the above solutions were stored at $4{ }^{\circ} \mathrm{C}$ until use. The calibration standards (CS) and quality control (QC) samples [lower limit of quantitation quality control, LLOQ QC (5.00 $\left.\mathrm{ng} \mathrm{mL}^{-1}\right)$; low quality control, LQC (15.0 $\left.\mathrm{ng} \mathrm{mL}^{-1}\right)$; medium quality control, MQC-2 (130.0 $\left.\mathrm{ng} \mathrm{mL}^{-1}\right)$ and MQC-1 (600.0 $\left.\mathrm{ng} \mathrm{mL}^{-1}\right)$; high quality control, HQC (1125 $\left.\mathrm{ng} \mathrm{mL} \mathrm{m}^{-1}\right)$; upper limit of quantitation quality control, ULOQ QC (1500 $\left.\mathrm{ng} \mathrm{mL}^{-1}\right)$ ] were prepared by spiking blank plasma with the respective working solutions (5\% of total plasma volume) in amber coloured ria vials (polypropylene tubes commonly used in radio immunoassays). CSs were made at 5.00, 10.0, 25.0, 50.0, 100.0, 200.0, 400.0, 800.0, 1200 and $1500 \mathrm{ng} \mathrm{mL}^{-1}$ for NFT. Spiked plasma samples were stored at $-20{ }^{\circ} \mathrm{C}$ for validation and subject sample analysis.

\section{Sample preparation procedure}

Prior to analysis, spiked plasma samples were thawed in a water bath and allowed to equilibrate at room temperature. The samples were adequately vortexed using a vor- 
texer before pipetting. Aliquots of $100 \mu \mathrm{L}$ plasma solution containing $5.0 \mu \mathrm{L}$ NFT working solution and $95 \mu \mathrm{L}$ blank plasma were transferred into amber coloured ria vials, $10 \mu \mathrm{L}$ of deionized water (for CS and QC samples) $/ 10 \mu \mathrm{L}$ of $50 \%$ DMF-deionized water solution (for study samples) was added and vortexed to mix. Further, $150 \mu \mathrm{L}$ IS working solution (1000 ng mL ${ }^{-1}$ ) and $25 \mu \mathrm{L}$ of $10 \mathrm{mmol} \mathrm{L}^{-1}$ ammonium acetate solution was added and vortexed again. Prior to loading plasma samples, Strata- $X$ cartridges were pre-washed with $1.0 \mathrm{~mL}$ of methanol, followed by $1.0 \mathrm{~mL}$ of deionized water and centrifuged after each step for $1.0 \mathrm{~min}$ at $1811 \times g$. Plasma samples were then applied to these conditioned cartridges and after centrifugation at $1811 \times g$ for $2.0 \mathrm{~min}$, washing was done with $1.0 \mathrm{~mL}$ of deionized water, followed by centrifugation for $1.0 \mathrm{~min}$ at $1811 \times \mathrm{g}$. Elution of samples was carried out with $1.0 \mathrm{~mL}$ of acetonitrile/deionized water $(60: 40, \mathrm{~V} / \mathrm{V})$, followed by centrifugation for $1.0 \mathrm{~min}$ at $1811 \times g$. The eluent was transferred to amber coloured auto injector vials and $5.0 \mu \mathrm{L}$ was used for injection in LC-MS/MS, in partial loop mode.

\section{Validation methodology}

A thorough and complete method validation of NFT in human plasma was done following the USFDA guidelines (15). Selectivity test was carried out in 13 different lots of blank human plasma including haemolysed and lipemic plasma collected with $\mathrm{K}_{3}$ EDTA as an anticoagulant. From each of these 13 different lots, two replicates of $95 \mu \mathrm{L}$ each were spiked with $5.0 \mu \mathrm{L}$ of the DMF/deionized water $(50: 50, V / V)$. In the first set, the double blank plasma (without analyte and IS) was directly injected after extraction, while the other set was spiked only with IS before extraction (total 26 samples). Further, one system suitability sample (SSS) at CS-2 (10.0 ng mL $\left.{ }^{-1}\right)$ concentration and two replicates of $L L O Q$ concentration were prepared by spiking $95 \mu \mathrm{L}$ blank human plasma with $5.0 \mu \mathrm{L}$ of the respective working aqueous standards of NFT. The acceptance criterion requires that at least $90 \%$ of selectivity samples should be free from any interference at the retention time of analyte and IS.

Interference due to commonly used medications in human volunteers was studied for acetaminophen, aspirin, caffeine, cetrizine, chlorpheniramine maleate, ibuprofen and pseudoephedrine. Their stock solutions $\left(100 \mu \mathrm{g} \mathrm{mL}^{-1}\right)$ were prepared by dissolving a requisite amount in methanol. Further, working solutions $\left(20.0 \mu \mathrm{g} \mathrm{mL}^{-1}\right)$ were prepared in $\mathrm{DMF} /$ deionized water (50:50, $V / V$ ), spiked in plasma (individually) along with NFT at LQC and HQC levels. The samples were prepared in triplicate and analyzed using the proposed extraction protocol. The final plasma concentration of these medications was $1000 \mathrm{ng} \mathrm{mL}^{-1}$. These sets were processed along with the freshly prepared calibration curve standards and two sets (8 samples) of qualifying QC samples (HQC, MQC-1, MQC-2 and LQC). As per the acceptance criteria, the accuracy should be within 85 and $115 \%$ (15).

A carryover experiment was performed to verify any carryover of analyte, which may be reflected in subsequent runs. The design of the study comprised the following sequence of injections, i.e., double blank plasma samples ${ }^{\circledR}$ two samples of $L L O Q{ }^{\circledR}$ double blank plasma samples ${ }^{\circledR} U L O Q$ sample ${ }^{\circledR}$ double blank plasma samples to check for any interference due to carryover.

The linearity of the method was determined by analysis of six calibration curves containing ten non-zero concentrations. The area ratio response for NFT/IS obtained from multiple monitoring was used for regression analysis. Each calibration curve was analyzed 
D. S. Patel et al.: Quantitation of nitrofurantoin in human plasma by liquid chromatography tandem mass spectrometry, Acta Pharm. 63 (2013) 141-158

individually using the least square weighted $\left(1 / x^{2}\right)$ linear regression. The coefficient of determination $\left(R^{2}\right)$ value $>0.99$ was desirable for all the calibration curves. The lowest standard on the calibration curve was accepted as the $L L O Q$, if the analyte response was at least ten times higher than that of the drug free (blank) extracted plasma.

Intra-batch and inter-batch (on three consecutive validation days) accuracy and precision were evaluated at six QC levels (LLOQ QC, LQC, MQC-2, MQC-1, HQC and ULOQ $\mathrm{QC}$ ) in six replicates for NFT. The deviation (RSD, \%) at each concentration level from the nominal concentration was expected to be within $\pm 15 \%$. Similarly, the mean accuracy should not vary by $\pm 15 \%$ except for the $L L O Q$ where it can be $\pm 20 \%$ of the nominal concentration. Also, 2/3 quality control samples should meet the nominal concentration criteria of $\pm 15 \%$. Reinjection reproducibility was performed by re-injecting one entire validation batch.

The relative recovery, matrix effect and process efficiency were evaluated as recommended by Matuszewski et al. (16) at HQC, MQC-1, MQC-2 and LQC levels with six replicates. Relative recovery (RE) was calculated by comparing the mean area response of samples spiked before extraction to that of samples spiked after extraction at each QC level. The recovery of IS was similarly estimated. Absolute matrix effect (ME) was assessed by comparing the mean area response of samples spiked after extraction with the mean area response of neat standard solutions prepared in elution solution. The overall process efficiency $(\mathrm{PE}, \%)$ was calculated as $(\mathrm{ME} \times \mathrm{RE}) / 100$. The relative matrix effect on analyte quantification was also checked in eight different batches/lots of plasma, which included six $\mathrm{K}_{3}$ EDTA and one each of haemolysed and lipemic plasma. From each of these batches, NFT samples at LQC and HQC levels were prepared in quadruplicate by spiking NFT in extracted blank plasma and checked for RSD (\%) and accuracy (\%). Deviation of the standards and QCs should not exceed $\pm 15 \%$.

Matrix ion suppression-enhancement effects on the MRM (full given earlier) LC-MS/ MS sensitivity were evaluated by the post column analyte infusion experiment (17). A standard solution containing NFT at the HQC level and $1000 \mathrm{ng} \mathrm{mL}^{-1}$ of IS in mobile phase was infused post column via a 'T' connector into the mobile phase at $5.0 \mu \mathrm{L} \mathrm{min}{ }^{-1}$ employing a Harvard infusion pump (Harvard Bioscience, USA). Aliquots of $5.0 \mu \mathrm{L}$ of extracted $1500 \mathrm{ng} \mathrm{mL}^{-1}$ sample and double blank plasma sample were then injected and MRM LC-MS/MS chromatograms were acquired for NFT and IS. Any dip in the baseline upon injection of double blank plasma would indicate ion suppression, while a peak at the retention time of NFT and IS indicated ion enhancement.

All stability results were evaluated by measuring the area ratio response (NFT/IS) of stability samples against freshly prepared comparison standards at LQC and HQC levels. Stock solutions of NFT and IS were checked for short term stability at room temperature and long term stability at $4{ }^{\circ} \mathrm{C}$. The solutions were considered stable if the deviation from the nominal value was within $\pm 10.0 \%$. Bench top stability, processed sample stability at room temperature and at refrigerated temperature $\left(4^{\circ} \mathrm{C}\right)$, freeze thaw stability and long term stability at $-20^{\circ} \mathrm{C}$ were assessed at LQC and HQC levels using six replicates at each level for NFT. To meet the acceptance criteria, the RSD (\%) and accuracy (\%) should be within $\pm 15 \%$. Also, at least $2 / 3$ quality control samples should meet the nominal concentration criteria of $\pm 15 \%$.

A dilution integrity experiment was performed with the aim to validate the dilution test to be carried out on higher analyte concentrations (above ULOQ), which may be en- 
countered during real subject samples analysis. Dilution integrity experiment for NFT was carried out using two solutions of different concentration, i.e., $7500 \mathrm{ng} \mathrm{mL}^{-1}$, which is 5 times the $U L O Q$ concentration $\left(1500 \mathrm{ng} \mathrm{mL}^{-1}\right.$ ) and $1125 \mathrm{ng} \mathrm{mL}^{-1}$ (HQC level). From each of these solutions, six replicate samples were prepared at $1 / 10^{\text {th }}$ dilution by taking $10 \mu \mathrm{L}$ and diluting it with $90 \mu \mathrm{L}$ screened blank human plasma. The precision and accuracy for these $1 / 10^{\text {th }}$ diluted standard solutions were determined by robustness analyzing the samples against freshly prepared calibration curve standards for NFT. Method was evaluated on two precision and accuracy batches. The first batch was analyzed by two analysts form different laboratories while the second batch was studied on two BDS Hypersil $\mathrm{C}_{18}$.

\section{Bioequivalence study and incurred sample reanalysis}

The study plan was an open label, randomized, two period, two treatment, two sequence, balanced, crossover, single dose evaluation of relative oral bioavailability of the test (100 mg nitrofurantoin macro-crystalline capsules of a generic company) and a reference (Macrodantin ${ }^{\circledR}, 100$ mg nitrofurantoin macro-crystalline capsules from Procter \& Gamble Pharmaceuticals, USA) formulation in 36 healthy subjects under fed conditions. All the subjects were informed of the aim and risk involved in the study and their written consents were obtained. The inclusion criteria for volunteer selection was based on the age (18-45 years), body mass index (between 18.5 and $24.9 \mathrm{~kg} \mathrm{~m}^{-2}$ ), general physical examination, electrocardiogram and laboratory tests like hematology, blood chemistry, urine examination and immunological tests. The exclusion criteria included allergic responses to NFT, volunteers with a history of alcoholism, smokers and those having a disease which may compromise the haematopoietic, gastrointestinal, renal, hepatic, cardiovascular, respiratory, central nervous system, diabetes, psychosis or any other body system. The work was subject to review and approved by an Independent Ethics Committee (IEC) of Cliantha Research Ltd., Ahmedabad, India. The subjects for the study were fasted $10 \mathrm{~h}$ before administration of the drug formulation. They were given high fat and high calory breakfast $30 \mathrm{~min}$ prior to taking the drug under investigation. Blood samples were collected in vacutainers containing $\mathrm{K}_{3}$ EDTA anticoagulant before $(0 \mathrm{~h})$ and at $0.5,1.0,2.0,3.0,3.5,4.0,4.5,5.0,5.5,6.0,6.5,7.0,8.0,10,12,16 \mathrm{~h}$ after drug administration. Blood samples were centrifuged at $2415 \times g$ at $4{ }^{\circ} \mathrm{C}$ for $15 \mathrm{~min}$; plasma was separated and stored at $-20{ }^{\circ} \mathrm{C}$ until use. The pharmacokinetic parameters of NFT were estimated by a non-compartmental model using the WinNonlin software version 5.2.1 (Pharsight Corporation, USA). An incurred sample re-analysis (ISR) was also conducted by computerized random selection of subject samples, $10 \%$ of total 1110 samples analyzed. The selection criteria included samples which were near the $c_{\max }$ and the elimination phase in the pharmacokinetic profile of the drug. The results obtained were compared with the data obtained earlier for the same sample using the same procedure. The percent change in the value should not be more than $\pm 20 \%$ (18). 
D. S. Patel et al.: Quantitation of nitrofurantoin in human plasma by liquid chromatography tandem mass spectrometry, Acta Pharm. 63 (2013) 141-158.

\author{
RESULTS AND DISCUSSION
}

\title{
Method development
}

The extraction procedure, mass spectrometry and chromatographic conditions were suitably optimized. During method development, electrospray ionization of NFT and nitrofurazone was conducted in positive as well as negative ionization mode using $500 \mathrm{ng} \mathrm{mL}-1$ tuning solution. However, the response was higher and consistent in the negative mode under MRM with a signal-to-noise ratio > 1000 (with validated/finalized integration parameters) and a good linearity in regression curves. The analyte and IS gave predominant singly charged deprotonated precursor $[\mathrm{M}-\mathrm{H}]^{-}$ions at $\mathrm{m} / \mathrm{z}$ of 237.0 and 197.0 for NFT and IS, respectively, in Q1 full scan spectra. Further, fragmentation was initiated using sufficient nitrogen for collision activation dissociation (CAD) and by applying $-17.0 \mathrm{~V}$ collision energy to break the precursor ions. The most abundant and consistent ion found in the product ion mass spectra of NLF was at $m / z 151.8$, resulting from the cleavage of the imidazolidine ring. The other characteristic fragment of NLF having nitrofuran substructure was observed at $\mathrm{m} / \mathrm{z} 123.9$, which can be attributed to the elimination of $\mathrm{N}_{2}$ from $m / z 151.8$, as shown in Fig. 1a. However, for nitrofurantoin, this fragment $(\mathrm{m} / \mathrm{z} 123.9)$ was the most abundant and reproducible product ion obtained at $15 \mathrm{~V}$ collision energy in the Q3 spectra. The other product ions obtained for IS were at $\mathrm{m} / \mathrm{z}$ 149.8 (due to elimination of nitro group from the precursor ion) and at $m / z 106.9$, as shown in Fig. 1b. To attain an ideal Taylor cone and a better impact on spectral response, an optimum potential of $-4500 \mathrm{~V}$ was kept, which gave a stable signal. Fine tuning of nebulizer gas (68947 Pa) and CAD gas was done to get a consistent and adequate response. The ion source chamber temperature had little effect on the signal and was hence maintained at $475{ }^{\circ} \mathrm{C}$. A dwell time of $300 \mathrm{~ms}$ was adequate and no crosstalk was observed between the MRMs of analytes.

During development, the chromatographic conditions were examined on BDS Hypersil C18, Hypurity C18 and Aquasil C18 (all $100 \times 4.6 \mathrm{~mm}, 5.0 \mu \mathrm{m}$ ) columns to achieve a short-run time, symmetric peak shapes, minimum matrix interference and solvent consumption. To find the best mobile phase, different volume ratios (70:30, 60:40, 50:50 and 40:60, $V / V$ ) of methanol/water and acetonitrile/water combinations were tried as mobile phases, along with formic acid (0.01-0.005\%) and ammonium acetate $\left(0.1-5 \mathrm{mmol} \mathrm{L}^{-1}\right)$. In addition, the effect of the flow rate was also studied from 0.3 to $1.0 \mathrm{~mL} \mathrm{~min}^{-1}$, which was also responsible for acceptable chromatographic peak shapes. Although acceptable chromatography was achieved on all three columns with symmetric peak shape, the run time was higher (> $5 \mathrm{~min}$ ) on the Hypurity C18 and Aquasil C18 compared to the BDS Hypersil C18 column. Moreover, the response was superior on the BDS Hypersil C18 column, especially at lower concentration ( $L L O Q$ and LQC) and hence it was selected for the present work. A chromatographic run time of $3.5 \mathrm{~min}$ was possible using a mobile phase consisting of acetonitrile $/ 0.25 \mathrm{mmol} \mathrm{L}^{-1}$ ammonium acetate $(60: 40, V / V)$. A flow rate of $0.5 \mathrm{~mL} \mathrm{~min}^{-1}$ was found most suitable for acceptable peak shapes and run time. The reproducibility of retention time for the analyte was $\leq 0.6 \%$ for 100 injections on the same column. The maximum on-column loading (at ULOQ) of NFT per sample injection volume was $0.75 \mathrm{ng}$. Due to the unavailability of deuterated analogue, a general 


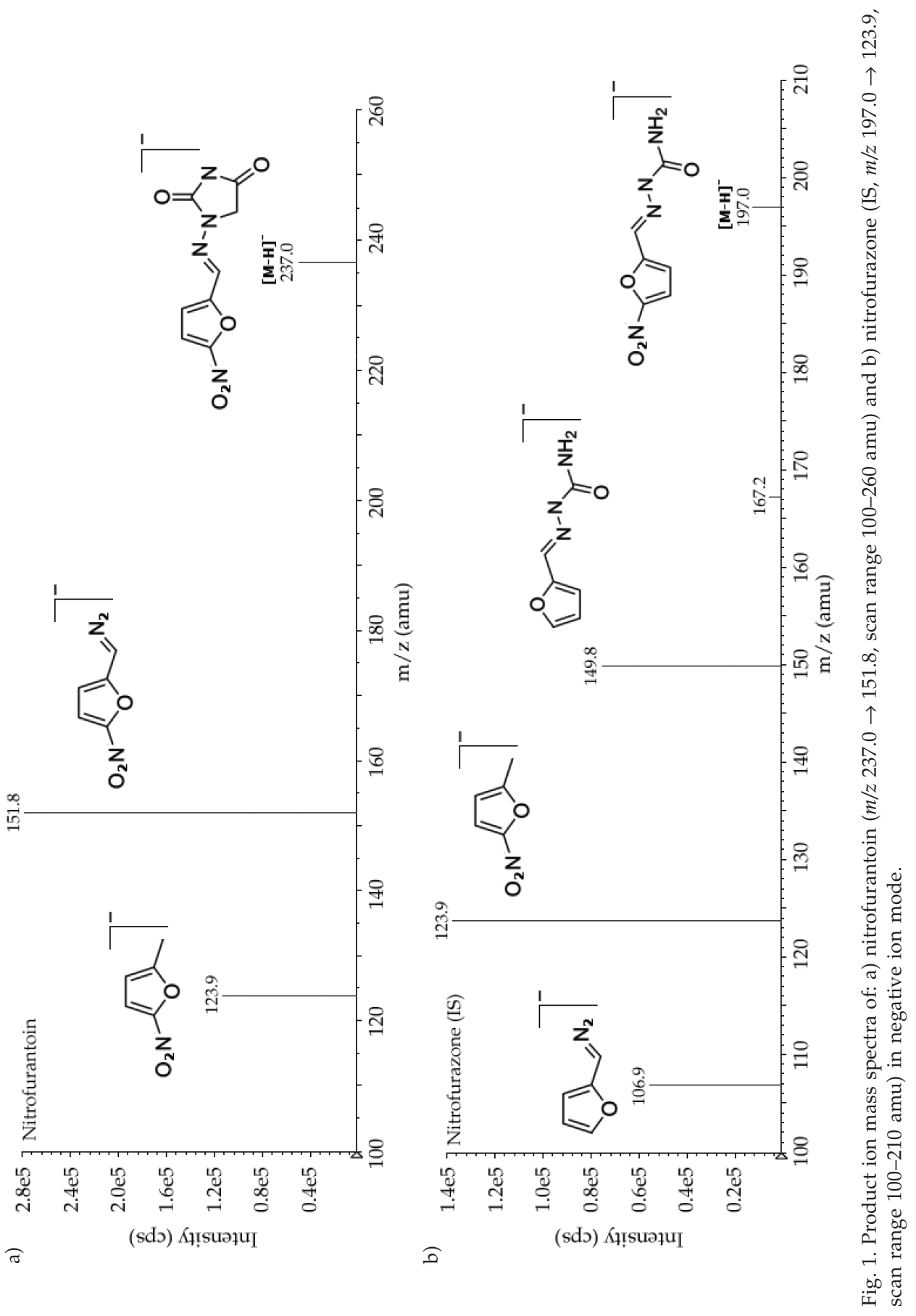


IS was used to minimize analytical variation due to solvent evaporation and ionization efficiency. Nitrofurazone, used as an IS in the present study is structurally similar to nitrofurantoin, both possessing a 5-nitrofuran ring with substitution at the 2-position. Both had similar chromatographic behavior and there was no effect of IS on analyte recovery, sensitivity or ion suppression.

All three conventional extraction techniques, namely, protein precipitation (PP), liquid-liquid extraction (LLE) and solid phase extraction (SPE), were tested in the present work for quantitative extraction of NFT from human plasma. PP was initially tried using methanol, acetonitrile and perchloric acid as precipitating agents but the response was inconsistent with some ion suppression at $L L O Q$ and LQC levels. LLE was carried out in ethyl acetate as reported earlier (10); however, the response found was inadequate at the LLOQ level.

Solid phase extraction was tried on Strata-X polymeric reversed cartridges employing $100 \mathrm{~mL}$ plasma for processing in the presence of $10 \mu \mathrm{mol} \mathrm{L} \mathrm{L}^{-1}$ ammonium acetate. Elution of samples with acetonitrile/deionized water $(60: 40, V / V)$ gave consistent and quantitative recovery (>91\%) for NFT and IS at all QC levels.

\section{System suitability, selectivity, interference and carryover check}

During method validation, precision (RSD, \%) was observed in the range of 0.1 to $0.6 \%$ for the retention time and from 1.6 to $2.9 \%$ for the area response of NFT and IS. All plasma samples studied for selectivity were found free from any endogenous interference. Figs. 2a-d demonstrate the selectivity results with chromatograms of double blank plasma (without IS), blank plasma (with IS), peak response of NFT at LLOQ concentration and a real subject sample chromatogram of NFT at $4.5 \mathrm{~h}$ after oral administration of $100 \mathrm{mg}$ capsule formulation. No interference was observed for commonly used medications like acetaminophen, aspirin, caffeine, cetrizine, chlorpheniramine maleate, ibuprofen and pseudoephedrine. Carryover evaluation was performed to ensure that it did not affect the accuracy and precision of the proposed method. Almost negligible area (less than $0.5 \%$ of $L L O Q$ area) was observed in double blank plasma run after $U L O Q$, which suggests no carryover of the analyte in subsequent runs.

\section{Linearity, sensitivity, accuracy and precision}

All six calibration curves were linear over the concentration range of $5.00-1500$ ng mL ${ }^{-1}$ with the correlation coefficient $R^{3} 0.9984$. A straight-line fit was made through the data points by least square regression analysis to give the mean linear equation $y=(0.0049 \pm 0.00011) \gamma+(0.0034 \pm 0.00013)$ where $y$ is the peak area ratio of the analyte / IS and $\gamma$ the concentration of the analyte. The accuracy and precision (RSD, \%) observed for the calibration curve standards ranged from 97.0 to $104.4 \%$ and 1.3 to $8.1 \%$, respectively. The lowest concentration $(L L O Q)$ in the standard curve that can be measured with acceptable accuracy and precision was $5.00 \mathrm{ng} \mathrm{mL}^{-1}$ at a signal-to-noise ratio $(S / N)^{3}$ 36 and a limit of detection $(L O D)$ of $0.25 \mathrm{ng} \mathrm{mL}^{-1}$.

The intra-batch and inter-batch precision and accuracy were established from validation runs performed at six QC levels (Table I). The intra-batch precision (RSD, \%) 


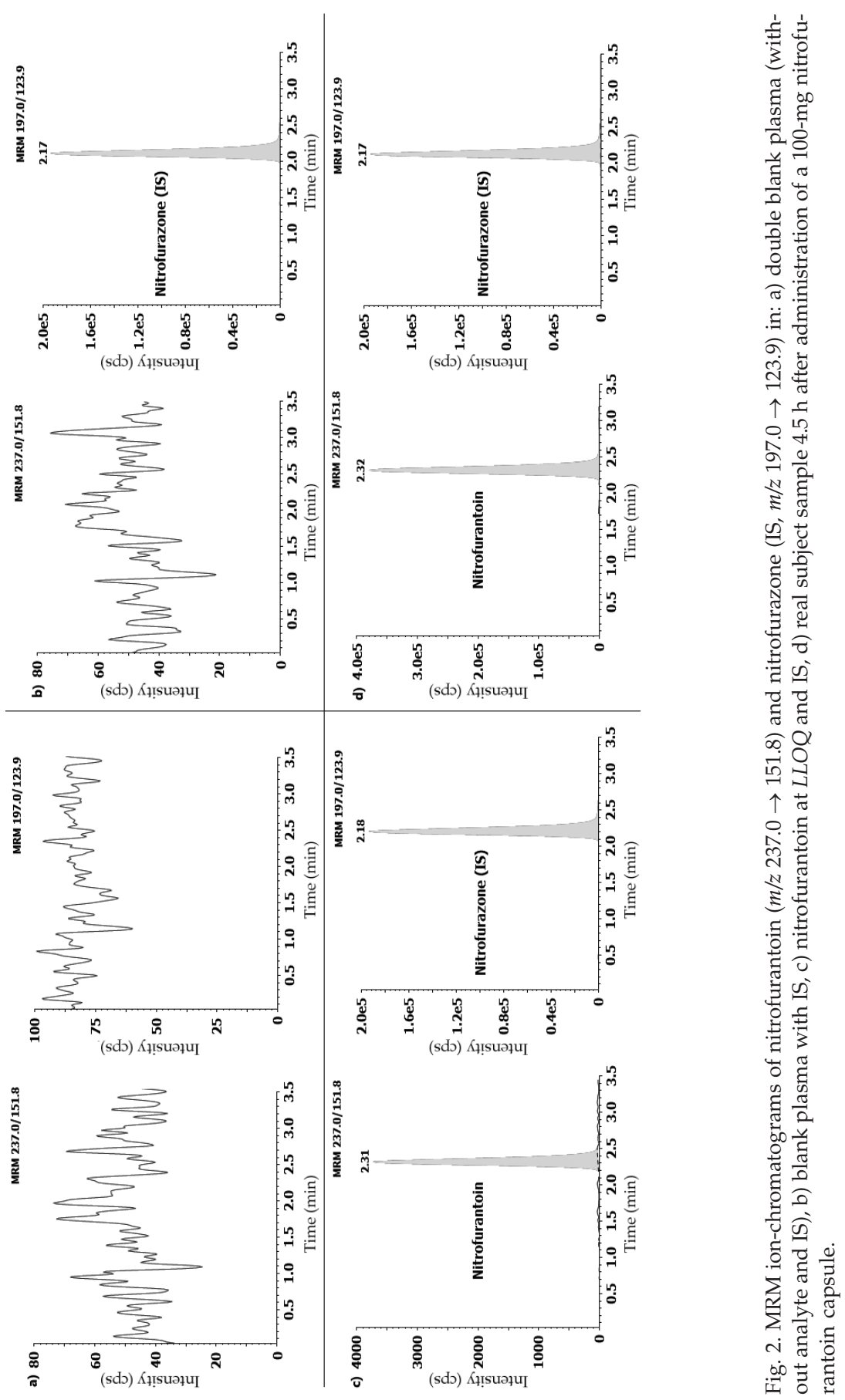


D. S. Patel et al.: Quantitation of nitrofurantoin in human plasma by liquid chromatography tandem mass spectrometry, Acta Pharm. 63 (2013) 141-158.

Table I. Intra-batch and inter-batch accuracy and precision for nitrofurantoin

\begin{tabular}{lccccccc}
\hline & \multicolumn{3}{c}{ Intra-batch } & \multicolumn{3}{c}{ Inter-batch } \\
\cline { 3 - 7 } QC ID & $\begin{array}{c}\text { Naded } \\
\text { (ng mL }\end{array}$ & $\begin{array}{c}\text { Mean conc. } \\
\text { found } \\
\left(\text { ng mL }^{-1}\right)^{\mathrm{a}}\end{array}$ & $\begin{array}{c}\text { Accuracy } \\
(\%)\end{array}$ & $\begin{array}{c}\text { RSD } \\
(\%)\end{array}$ & $\begin{array}{c}\text { Mean conc. } \\
\text { found } \\
\left(\text { ng mL }^{-1}\right)^{\mathrm{b}}\end{array}$ & $\begin{array}{c}\text { Accuracy } \\
(\%)\end{array}$ & $\begin{array}{c}\text { RSD } \\
(\%)\end{array}$ \\
\hline LLOQ & 5.00 & 5.05 & 101.0 & 2.2 & 4.76 & 95.2 & 5.0 \\
LQC & 15.0 & 14.9 & 99.3 & 3.6 & 14.7 & 98.0 & 4.3 \\
MQC-2 & 130.0 & 129.1 & 99.3 & 3.0 & 127.5 & 98.1 & 3.0 \\
MQC-1 & 600.0 & 582.4 & 97.1 & 5.2 & 591.4 & 98.6 & 5.8 \\
HQC & 1125.0 & 1194.1 & 106.1 & 2.4 & 1132.2 & 100.6 & 5.2 \\
ULOQ & 1500.0 & 1495.9 & 99.7 & 1.2 & 1414.1 & 94.3 & 5.5 \\
\hline
\end{tabular}

a Mean of six replicate observations at each concentration.

$\mathrm{b}$ Mean of eighteen replicate observations over three different analytical runs.

ranged from 1.2 to $5.2 \%$ and the accuracy was within 97.1 and $105.1 \%$. For the inter-batch experiments, the precision varied from 3.0 to 5.8 and the accuracy was within 94.3 and $100.6 \%$.

\section{Recovery, matrix effect, matrix factor, ion suppression}

The relative recovery, absolute matrix effect and process efficiency data for NFT are presented in Table II. The relative recovery of the analyte is 'true recovery', which is unaffected by the matrix as it is calculated by comparing the area ratio response (analyte/IS) of extracted (spiked before extraction) and unextracted (spiked after extraction) samples. The relative recovery obtained for NFT and IS was > $91 \%$ at all QC levels. Further, the relative matrix effect which compares the precision values (RSD, \%) between different lots (sources) of plasma (spiked after extraction) samples varied from 0.9 to 2.5 for NFT at the LQC and HQC levels. The accuracy values ranged from 98.7 to $103.1 \%$ for both concentration levels. Results of the post-column analyte infusion chromatogram in Fig. 3 indicate no ion suppression or enhancement at the retention time of NFT and IS. A minor suppression in the response was observed at 1.2 min; however, it did not interfere with the quantitation. The average matrix factor value calculated as the response of post spiked sample/response of neat solution (in elution solution) at the $L L O Q$ level was 0.98 , which indicates a minor suppression of about $2 \%$.

\section{Stability and dilution integrity}

Stability experiments were performed to evaluate analyte stability in stock solutions and in plasma samples under different conditions, simulating the same conditions that occurred during study sample analysis. The stock solution of NFT was stable at room temperature for $6 \mathrm{~h}$ and at $4{ }^{\circ} \mathrm{C}$ for 17 days. The intermediate stock solution of NFT in $\mathrm{DMF} /$ deionized water $(50: 50, \mathrm{~V} / \mathrm{V})$ was stable at $4{ }^{\circ} \mathrm{C}$ for 14 days with a change of $-0.1 \%$. 
D. S. Patel et al:: Quantitation of nitrofurantoin in human plasma by liquid chromatography tandem mass spectrometry, Acta Pharm. 63 (2013) 141-158.

Table II. Absolute matrix effect, relative recovery and process efficiency for nitrofurantoin

\begin{tabular}{|c|c|c|c|c|c|}
\hline $\begin{array}{c}\mathrm{A} \\
(\mathrm{RSD}, \%)\end{array}$ & $\begin{array}{c}\text { B } \\
(\mathrm{RSD}, \%)\end{array}$ & $\begin{array}{c}\mathrm{C} \\
(\mathrm{RSD}, \%)\end{array}$ & $\begin{array}{c}\text { Absolute matrix } \\
\text { effect }(\%)^{\mathrm{a}}\end{array}$ & $\begin{array}{c}\text { Relative } \\
\text { recovery }(\%)^{\mathrm{b}}\end{array}$ & $\begin{array}{c}\text { Process } \\
\text { efficiency }(\%)^{c}\end{array}$ \\
\hline \multicolumn{6}{|c|}{ LQC } \\
\hline $98963(3.2)$ & $96267(2.5)$ & $89764(2.9)$ & $97.3(100.8)^{\mathrm{d}}$ & $93.2(93.6)^{\mathrm{d}}$ & $90.7(94.3)^{\mathrm{d}}$ \\
\hline \multicolumn{6}{|c|}{ MQC-2 } \\
\hline $821457(2.8)$ & $801835(0.7)$ & $760287(2.5)$ & $97.6(101.7)^{\mathrm{d}}$ & $94.8(92.7)^{\mathrm{d}}$ & $92.5(94.3)^{\mathrm{d}}$ \\
\hline \multicolumn{6}{|c|}{ MQC-1 } \\
\hline $3858520(1.8)$ & $3696712(1.8)$ & $3383058(2.6)$ & $95.8(102.5)^{\mathrm{d}}$ & $91.5(92.2)^{\mathrm{d}}$ & $87.7(94.5)^{\mathrm{d}}$ \\
\hline \multicolumn{6}{|c|}{ HQC } \\
\hline 7481403 (2.0) & 7183531 (1.5) & $6685993(2.8)$ & $96.0(100.2)^{\mathrm{d}}$ & $93.1(91.4)^{\mathrm{d}}$ & $89.4(91.6)^{\mathrm{d}}$ \\
\hline
\end{tabular}

A - Mean area response of six replicate samples prepared in mobile phase (neat samples).

B - Mean area response of six replicate samples prepared by spiking in extracted blank plasma.

$\mathrm{C}-$ Mean area response of six replicate samples prepared by spiking before extraction.

${ }^{\mathrm{a}} \mathrm{B} / \mathrm{A} \times 100$

$\mathrm{b} C / \mathrm{B} \times 100$

${ }^{\mathrm{c}} \mathrm{C} / \mathrm{A} \times 100$

${ }^{d}$ In parentheses values for internal standard, nitrofurazone.
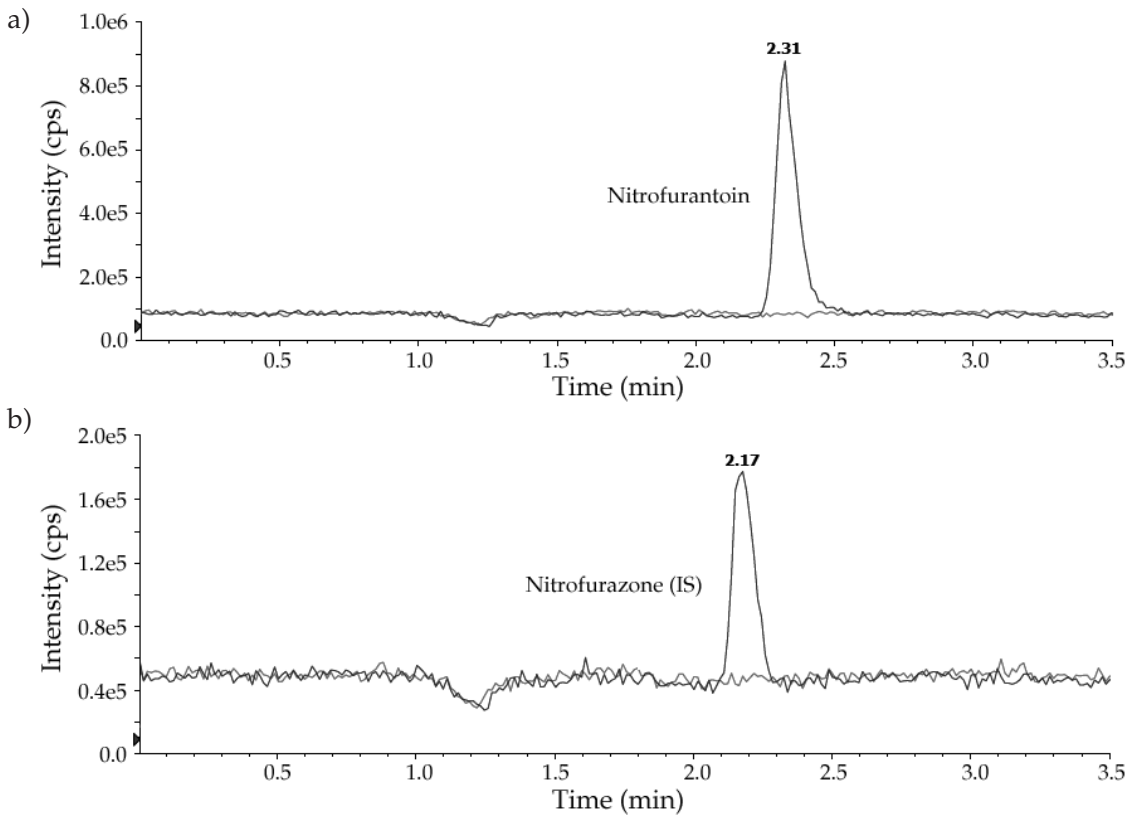

Fig. 3. Representative post column analyte infusion MRM LC-MS/MS overlaid chromatograms for nitrofurantoin and nitrofurazone: a) exact ion current (XIC) chromatogram of nitrofurantoin $(\mathrm{m} / \mathrm{z}$ $237.0 \rightarrow 151.8$ ), b) XIC of nitrofurazone (IS, $m / z$ 197.0 $\rightarrow$ 123.9). 
NFT was found stable in control plasma at room temperature up to $24 \mathrm{~h}$ and for six freeze and thaw cycles. The analyte in extracted plasma samples (processed samples) in acetonitrile/deionized water $(60: 40, V / V)$ was stable for $98 \mathrm{~h}$ under refrigerated conditions (4 ${ }^{\circ} \mathrm{C}$ ) and for $98 \mathrm{~h}$ at room temperature. Spiked plasma samples of NFT stored at $-20{ }^{\circ} \mathrm{C}$ for long term stability were found stable for a minimum period of 95 days. Values for the percent change for all stability experiments are compiled in Table III.

The precision values for dilution integrity of $1 / 10$ of 5 times ULOQ $\left(750.0 \mathrm{ng} \mathrm{mL}^{-1}\right)$ and $1 / 10$ of HQC (112.5 $\left.\mathrm{ng} \mathrm{mL}^{-1}\right)$ concentration were 1.3 and $0.9 \%$, while accuracy results were within 94.8 and $97.6 \%$, respectively, which is within the acceptance limit of $15 \%$ for precision and 85 to $115 \%$ for accuracy. For method robustness the precision and accuracy values for two different columns ranged from 3.0 to $3.8 \%$ and 96.5 to $101.2 \%$, respectively, at all six QC levels. For the experiment with different analysts, the results for precision and accuracy were within 2.2 to $3.3 \%$ and 97.2 to $102.5 \%$, respectively, at these levels.

\section{Application of the method to human subjects and incurred sample reanalysis}

The validated method was applied for a bioequivalence study of NFT in 36 healthy male subjects, who received $100 \mathrm{mg}$ test and reference formulations of NFT under fed conditions. The mean pharmacokinetic profile for the treatment is presented in Fig. 4. The method was sensitive enough to monitor their plasma concentration up to $16 \mathrm{~h}$. In all, 1968 samples including calibration, QC and volunteer samples were run and analyzed. The precision and accuracy for calibration and QC samples were well within the acceptable limits. The mean pharmacokinetic parameters obtained for the test and reference for-

Table III. Stability results for nitrofurantoin under different conditions

\begin{tabular}{|c|c|c|c|c|}
\hline Stability & Storage condition & Level & $\begin{array}{c}\text { Stability of the } \\
\left.\text { sample (ng mL } \mathrm{mL}^{-1}\right)^{\mathrm{a}}\end{array}$ & Change $(\%)^{b}$ \\
\hline \multirow{2}{*}{$\begin{array}{l}\text { Bench top } \\
\text { stability }\end{array}$} & \multirow{2}{*}{$\begin{array}{l}\text { Room temperature } \\
(24 \mathrm{~h})\end{array}$} & LQC & $13.71 \pm 0.72$ & -8.7 \\
\hline & & HQC & $1131.03 \pm 23.37$ & 0.5 \\
\hline \multirow{2}{*}{$\begin{array}{l}\text { Processed sample } \\
\text { stability (extracted } \\
\text { samples) }\end{array}$} & \multirow{2}{*}{$\begin{array}{l}\text { Auto sampler } \\
\left(4^{\circ} \mathrm{C}, 98 \mathrm{~h}\right)\end{array}$} & LQC & $13.70 \pm 0.34$ & -8.7 \\
\hline & & HQC & $1102.14 \pm 22.39$ & -2.0 \\
\hline \multirow{2}{*}{$\begin{array}{l}\text { Processed sample } \\
\text { stability (extracted } \\
\text { samples) }\end{array}$} & \multirow{2}{*}{$\begin{array}{l}\text { Room temperature } \\
(98 \mathrm{~h})\end{array}$} & LQC & $14.33 \pm 0.15$ & -4.6 \\
\hline & & HQC & $1083.23 \pm 20.53$ & -3.7 \\
\hline \multirow{2}{*}{$\begin{array}{l}\text { Freeze and thaw } \\
\text { stability }\end{array}$} & \multirow{2}{*}{$\begin{array}{l}\text { After } 6^{\text {th }} \text { cycle at } \\
-20^{\circ} \mathrm{C}\end{array}$} & LQC & $14.13 \pm 0.21$ & -6.0 \\
\hline & & $\mathrm{HQC}$ & $1064.12 \pm 18.28$ & -5.4 \\
\hline \multirow{2}{*}{ Long term stability } & \multirow{2}{*}{$\begin{array}{l}95 \text { days at } \\
-20^{\circ} \mathrm{C}\end{array}$} & LQC & $15.51 \pm 0.63$ & 3.3 \\
\hline & & HQC & $1038.42 \pm 27.23$ & -7.7 \\
\hline
\end{tabular}

LQC - low quality control (15 $\left.\mathrm{ng} \mathrm{mL}^{-1}\right)$.

$\mathrm{HQC}$ - high quality control (1125 $\left.\mathrm{ng} \mathrm{mL}^{-1}\right)$.

a Mean \pm SD, $n=6$ (replicates at each level).

b Values within the acceptance criteria of $\pm 15 \%$ as per US FDA guidelines (15). 


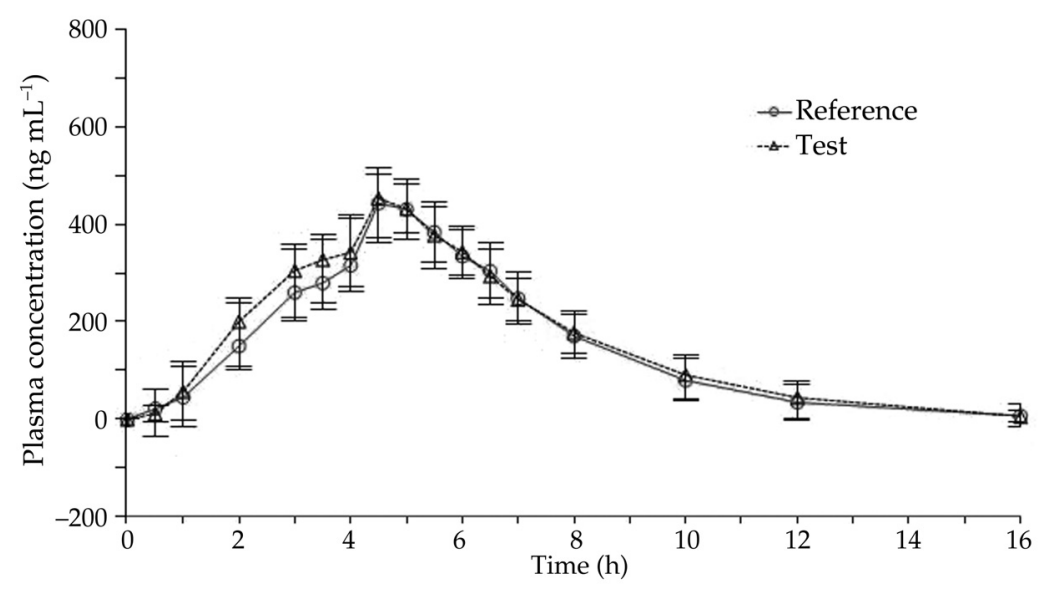

Fig. 4. Mean plasma concentration-time profile of nitrofurantoin after oral administration of a test (100 mg, nitrofurantoin capsules from a generic company) and a reference (100 mg, nitrofurantoin capsules from Procter \& Gamble Pharmaceuticals, USA) formulation to 36 healthy subjects under fed condition. Mean $\pm \mathrm{SD}, n=36$.

mulation are presented in Table IV. The $c_{\max }$ and $t_{\max }$ values obtained under fed conditions were slightly higher and lower, respectively, compared to the reported work (13). This may be due to genetic difference, race, age, gender (body size and muscle mass), type of food, etc., which may result in pharmacokinetic differences. However, their $A U C_{0 \text {-inf }}$ value

Table IV. Mean pharmacokinetic parameters after administration of a 100-mg nitrofurantoin capsule formulation administrated orally to 36 healthy subjects under fed conditions.

\begin{tabular}{ccc}
\hline Parameter & Reference & Test \\
\cline { 2 - 3 } & Mean $\pm \mathrm{SD}^{\mathrm{a}}$ & Mean $\pm \mathrm{SD}^{\mathrm{a}}$ \\
\hline$c_{\max }\left(\mathrm{ng} \mathrm{mL} \mathrm{m}^{-1}\right)$ & $513.4 \pm 49.0$ & $506.5 \pm 37.2$ \\
$t_{\max }(\mathrm{h})$ & $4.50 \pm 1.04$ & $4.71 \pm 1.05$ \\
$t_{1 / 2}(\mathrm{~h})$ & $1.66 \pm 0.31$ & $1.55 \pm 0.32$ \\
$A U C_{0-16 \mathrm{~h}}(\mathrm{~h} \mathrm{ng} \mathrm{mL}$ & $2401.65 \pm 405.34$ \\
$A U C_{0-\text { inf }}\left(\mathrm{h} \mathrm{ng} \mathrm{mL}^{-1}\right)$ & $2321.58 \pm 477.19$ & $2563.89 \pm 425.28$ \\
$k_{\mathrm{el}}\left(\mathrm{h}^{-1}\right)$ & $2622.29 \pm 534.61$ & $0.447 \pm 0.019$ \\
\hline
\end{tabular}

$c_{\max }-$ maximum plasma concentration.

$t_{\max }$ - time point of maximum plasma concentration.

$t_{1 / 2}$ - half-life of drug elimination during the terminal phase.

$A U C_{0-t}$ - area under the plasma concentration-time curve from zero hour to $16 \mathrm{~h}$.

$A U C_{0 \text {-inf }}$ - area under the plasma concentration-time curve from zero hour to infinity.

$k_{\mathrm{el}}$ - elimination rate constant.

a $n=36$ 


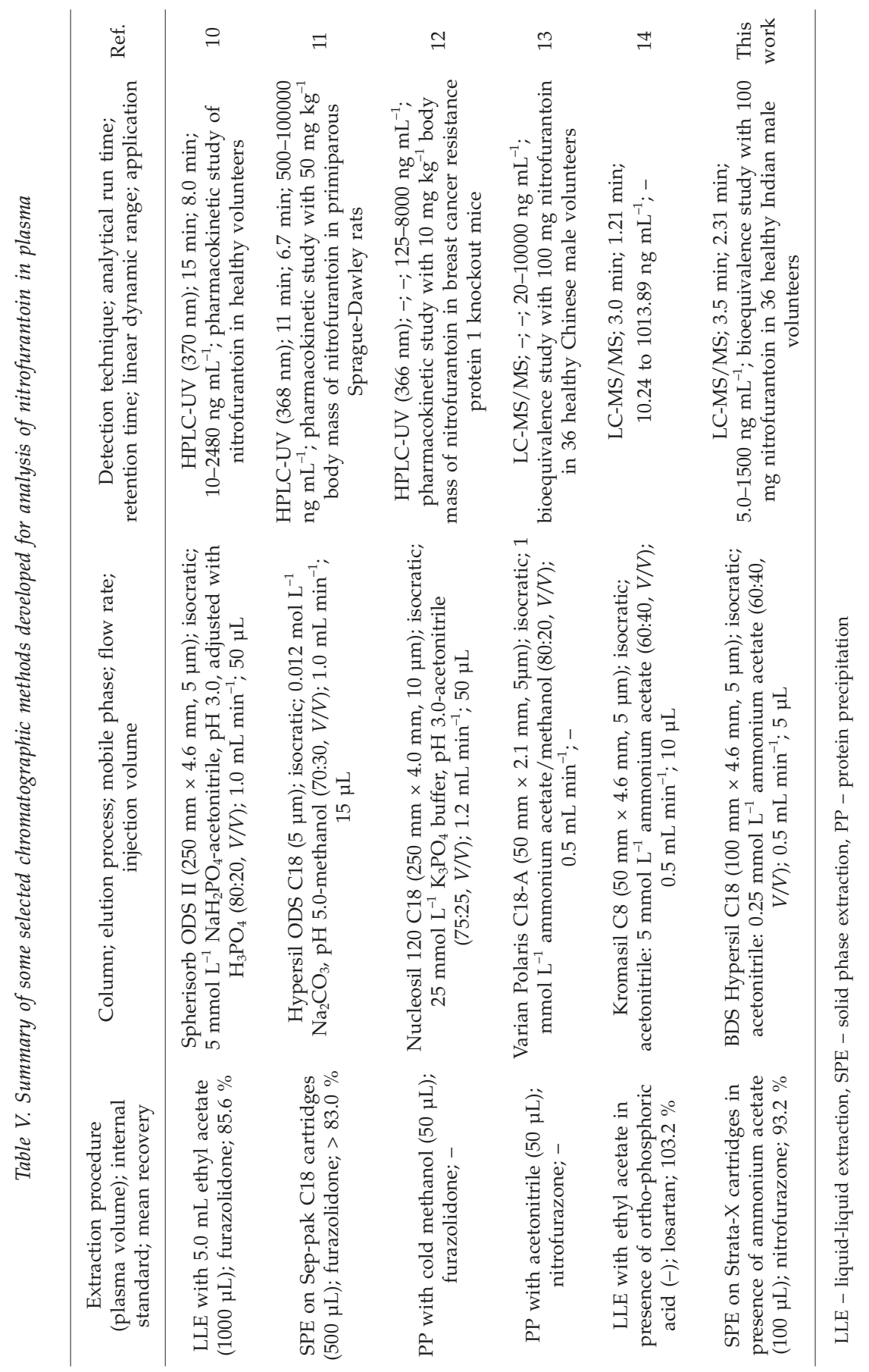


was comparable with the present work. The ratio of mean log-transformed data (test/reference formulation) for $c_{\max }, A U C_{0-\mathrm{t}}$ and $A U C_{0 \text {-inf }}(98.7,103.5$ and $97.8 \%$, respectively) and their $90 \%$ confidence intervals (93.1-103.1, 98.0-108.1 and 92.6-102.5\%, respectively) were all within the defined bioequivalence range. These observations confirm the bioequivalence of the test sample with the reference product in terms of rate and extent of absorption. Out of 111 reanalyzed incurred samples, 83 samples showed a change for assay reproducibility within $\pm 5 \%$ while the remaining 28 samples were within \pm 5.0 to $\pm 10 \%$. This authenticates the reproducibility of the proposed method.

\section{Comparison with reported methods}

The method presented has a higher sensitivity compared to previously reported methods (9-14) developed for NFT in plasma. The organic solvent consumption per sample analysis (extraction and chromatography) was only $2.7 \mathrm{~mL}$, which is considerably lower compared to these methods. Ion suppression/enhancement was checked by the post-column infusion technique and the quantitative assessment of matrix effects was evaluated by calculating the absolute and differential matrix effect, which was not reported in previous studies. Similarly, incurred sample reanalysis, which has now become mandatory for clinical and non-clinical studies, has proven the reproducibility in the measurement of subject samples for the first time for NFT. Table V gives a comparison of the salient features of chromatographic methods developed for NFT in plasma.

\section{CONCLUSIONS}

The objective of the present work was to develop and fully validate a simple, selective and sensitive method for NFT in human plasma by the turbo ion spray LC-MS/MS for routine sample analysis. The solid phase extraction procedure employed in the present work gave consistent and quantitative recoveries of NFT at all QC levels. The method requires only $100 \mu \mathrm{L}$ human plasma for extraction and demonstrates excellent performance in terms of robustness and efficiency (3.5 min per sample). The dynamic linear range was validated from $5.0-1500 \mathrm{ng} \mathrm{mL}^{-1}$. The maximum on-column loading at $U L O Q$ was $0.75 \mathrm{ng}$ for a $5.0 \mu \mathrm{L}$ injection volume, which helps in maintaining the efficiency and lifetime of the column. Moreover, the limit of quantification is low enough to monitor at least five half-lives of NFT concentration with good intra- and inter-assay reproducibility for quality control. The method was selective in the presence of medications commonly used by human volunteers. Ion suppression/enhancement was studied by the post column infusion of analyte and post extraction spiking technique. The proposed method has been successfully applied to a bioequivalence study of $100 \mathrm{mg}$ nitrofurantoin capsule formulation in 36 healthy males under fed condition. Reliability of the measurement of study samples is shown by incurred sample reanalysis.

Acknowledgements. - The authors are indebted to Mr. Vijay Patel, Executive Director, Mr. Anshul Dogra, Study Director and Mrs. Arpana Prasad, R\&D Supervisor, Cliantha Research Limited, Ahmedabad, for their continuous support, motivation and assistance during the course of this project. 


\section{REFERENCES}

1. D. R. Guay, An update on the role of nitrofurans in the management of urinary tract infections, Drugs 61 (2001) 353-364.

2. D. C. Hooper, Urinary Tract Agents: Nitrofurantoin and Methenamine, in Principles and Practice of Infectious Diseases (Eds. G. L. Mandell, J. E. Bennet and R. Dolin), Elsevier Churchill Livingstone Inc., Philadelphia 2009, pp. 515-520.

3. M. Maaland, and L. Guardabassi, In vitro antimicrobial activity of nitrofurantoin against Escherichia coli and Staphylococcus pseudintermedius isolated from dogs and cats, Vet. Microbiol. 151 (2011) 396-399; DOI: 10.1016/j.vetmic.2011.03.009.

4. AHFS Drug Information (Ed. G. K. Mcevoy), American Society of Hospital Pharmacists, Betuerda (MD) 1990, p. 438.

5. A. Bains, D. Buna and N. A. Hoag, A retrospective review assessing the efficacy and safety of nitrofurantoin in renal impairment, Can. Pharm. J. 142 (2009) 248-252; DOI: 10.3821/1913 -701X-142.5 .248 .

6. W. A. Petri, Sulfonamides, trimethoprim, sulfamethoxazole, quinolones, and agents for urinary tract infections, in Goodman \& Gilman's Pharmacological Basis of Therapeutics (Eds. L. L. Brunton, J. S. Lazo and K. L. Parker), McGraw Hill, New York, 2006, pp. 1111-1125.

7. M. Khodari, H. Mansour, H. S. El-Din and G. Mersal, Cathodic stripping voltammetry of the antibacterial drug (nitrofurantoin), Anal. Lett. 31 (1998) 251-262; DOI: 10.1080/00032719 808002043.

8. E. Hammam, Determination of nitrofurantoin drug in pharmaceutical formulation and biological fluids by square-wave cathodic adsorptive stripping voltammetry, J. Pharm. Biomed. Anal. 30 (2002) 651-659; DOI: 10.1016/S0731-7085(02)00344-8.

9. V. Arancibia, M. Valderrama, A. Madariaga, M. C. Zúñiga and R. Segura, Extraction of nitrofurantoin and its toxic metabolite from urine by supercritical fluids. Quantitation by high performance liquid chromatography with UV detection, Talanta 61 (2003) 377-383; DOI: 10.1016/ S0039-9140(03)00276-5.

10. P. Muth, R. Metz, B. Siems, W. W. Bolten and H. Vergin, Sensitive determination of nitrofurantoin in human plasma and urine by high-performance liquid chromatography, J. Chromatogr. A 729 (1996) 251-258; DOI: 10.1016/0021-9673(95)00894-2.

11. F. W. Kari, R. Weaver and M. C. Neville, Active transport of nitrofurantoin across the mammary epithelium in vivo, J. Pharmacol. Exp. Ther. 280 (1997) 664-668; DOI: 0022-3565/97/2802-0664.

12. G. Merino, J. W. Jonker, E. Wagenaar, A. E. van Herwaarden and A. H. Schinkel, The breast cancer resistance protein (BCRP/ABCG2) affects pharmacokinetics, hepatobiliary excretion, and milk secretion of the antibiotic nitrofurantoin, Mol. Pharmacol. 67 (2005) 1758-1764; DOI: 10.1124/ mol.104.010439.

13. K. K. Adkison, S. S. Vaidya, D. Y. Lee, S. H. Koo, L. Li, A. A. Mehta, A. S. Gross, J. W. Polli, Y. Lou and E. J. Lee, The ABCG2 C421A polymorphism does not affect oral nitrofurantoin pharmacokinetics in healthy Chinese male subjects, Br. J. Clin. Pharmacol. 66 (2008) 233-239; DOI: 10.1111/j.1365-2125.2008.03184.x.

14. R. S. Patil, K. A. Chaitanya, P. V. D. L. S. Ravi Prakash and S. R. Patil, Simple, rapid and sensitive method for determination of nitrofurantoin in human plasma by using liquid chromatography/ tandem mass spectrometry, Curr. Trends Biotechnol. Pharm. 6 (2012) 196-203.

15. US Department of Health and Human Services, Food and Drug Administration Centre for Drug Evaluation and Research, Guidance for Industry, Bioanalytical Method Validation, CDER, Centre for Veterinary Medicine (CVM), Rockville (MD) 2001. 
D. S. Patel et al.: Quantitation of nitrofurantoin in human plasma by liquid chromatography tandem mass spectrometry, Acta Pharm. 63 (2013) 141-158.

16. B. K. Matuszewski, M. L. Constanzer and C. M. Chavez-Eng, Strategies for the assessment of matrix effect in quantitative bioanalytical methods based on HPLC-MS/MS, Anal. Chem. 75 (2003) 3019-3030; DOI: 10.1021/ac020361s.

17. R. King, R. Bonfiglio, C. Fernandez-Metzler, C. Miller-Stein and T Olah, Mechanistic investigation of ionization suppression in electrospray ionization, J. Am. Soc. Mass Spectrom. 11 (2000) 942-950; DOI: 10.1016/S1044-0305(00)00163-X.

18. M. Yadav, and P. S. Shrivastav, Incurred sample reanalysis: A decisive tool in bioanalytical research, Bioanalysis 3 (2011) 1007-1024; DOI: 10.4155/bio.11.76. 